\title{
Using differential transform method to solve fractional non- linear integro-differential equations
}

\author{
L. Noor Ali Hussein \\ Department of Mathematics, Education of College , Al-Qadisiyah \\ University
}

\begin{abstract}
In this paper we'll to solve the fractional integro-differential equations by employment differential transform method and compare with integro-differential equations by graph.
\end{abstract}

\section{1- Introduction}

In this paper we'll find solution the fractional non-linear integro-

differential equations which the form $v^{q}(t)=$ $\left(t, v(t), v^{\prime}(t), \int_{t_{0}}^{t} G\left(s, v(s), v^{\prime}(s)\right) d s\right.$,

with conditians

$v\left(t_{0}\right)=v_{0}, v^{\prime}\left(t_{0}\right)=v_{1}$.

Where $t \in\left[t_{0}, T\right]$ and $m-1 \leq q \leq$ $m, m \in N$,

by using differential transform method

There are sundry definitions of a fractional derivativ of order $q>0$, here we depended on Caputo definition.

$D_{t_{0}}^{q} f(t)=J^{m-q}\left[\frac{d^{m}}{d t^{m}} f(t)\right]$

Where $m-1<q \leq m$ and $m \in N$.

The Caputo fractional drivative first calculates an ordnary drivative followed by a fractional integral to ascertain the wanted order of fractional derivative .

\section{2- Differential Transform}

Definition 2.1. Let $z(t)$, is anatomy function of one inconstant which is defined on $L=[0, t] \subseteq \Re$ and $t_{0} \in L$. $Z(k)$, is Differential transform of $z(t)$ and is predefined on $N$ union $\{0\}$ as the following: 
$Z(k)=\frac{1}{k !}\left[\frac{d^{k} z(t)}{d t^{k}}\right]_{t=t_{0}}$

where $z(t)$ is the fundamental function and $Z(k)$ is called the transformed function .Inverse differential transform of $Z(k)$ in the is predefined as follows

$$
z(t)=\sum_{k=0}^{\infty} Z(k)\left(t-t_{0}\right)^{k} .
$$

Then from the above two equations (4)and (5), with $t_{0}=0$, the function $z(t)$ can be written as:

$z(t)=\sum_{k=0}^{\infty} \frac{1}{k !}\left[\frac{d^{k} z(t)}{d t^{k}}\right]_{t=0} t^{k}$

the principal mathematical specifications of differential transform can be summarized in the following theorems .

\section{Theorems [1],[2]}

\section{Theorem 3.1}

If $Z(k), F(k)$ and $G(k)$ are differential transforms of the functions $z(t), f(t)$ and $g(t)$ consecutive, then :

1. If $z(t)=f(t) \pm g(t)$ then $Z(k)=F(k) \pm G(k)$.

2. If $z(t)=a f(t)$ then $Z(k)=$ $a F(k)$.

3. If $z(t)=f(t) g(t)$ then $Z(k)=$ $\sum_{l=1}^{k} F(l) G(k-l)$.

4. If $z(t)=\frac{d f(t)}{d t}$ then $Z(k)=$ $(k+1) F(k+1)$.
5. If $z(t)=\frac{d^{m} f(t)}{d t^{m}}$ then $Z(k)=(k+$ 1) $(k+2) \cdots(k+m) F(k+m)$.

6. If $z(t)=\int_{0}^{t} f(s) d s$ then $Z(k)=$ $\frac{F(k-1)}{k}, K \geq 1, Z(0)=0$.

7. If $z(t)=t^{m}$ then $Z(k)=$ $\delta(k-m)= \begin{cases}1, & k=m \\ 0, & O . W .\end{cases}$

8. If $z(t)=\sin (\omega t+a)$ then $Z(k)=\frac{\omega^{k}}{k !} \sin \left(\frac{k \pi}{2}+a\right)$.

9. If $z(t)=\cos (\omega t+a)$ then $Z(k)=\frac{\omega^{k}}{k !} \cos \left(\frac{k \pi}{2}+a\right)$.

10. If $z(t)=e^{\omega t}$ then $Z(k)=\frac{\omega^{k}}{k !}$.

Theorem 3.2. Assume that $Z(k), W(k), J_{1}(k)$ and $J_{2}(k)$, are the differential transforms of the functions $z(t), w(t), j_{1}(t)$ and $j_{2}(t)$, consecutive, then for $k=1,2, \cdots, N$,

1. If $z(t)=\int_{t_{0}}^{t} j_{1}(s) j_{2}(s) d s$ then $Z(k)=\frac{1}{k} \sum_{\ell=0}^{k-1} J_{1}(\ell) J_{2}(k-\ell-1)$

2. If $z(t)=w(t) \int_{t_{0}}^{t} j_{1}(s) j_{2}(s) d s$ then

$Z(k)=$

$\sum_{\ell=0}^{k} \sum_{s=0}^{k-\ell-1} \frac{1}{k-\ell} W(\ell) J_{1}(s) J_{2}(k-\ell-$ $s-1)$.

3. If $z(t)=\int_{t_{0}}^{t} \frac{d^{n_{1}}}{d t^{n_{1}}} j_{1}(s) \frac{d^{n_{2}}}{d t^{n_{2}}} j_{2}(s) d s$, then

$Z(k)=\frac{1}{k} \sum_{\ell=0}^{k-1} \frac{\left(n_{1}+\ell\right) !\left(n_{2}+k-\ell-1\right) !}{l !(k-\ell-1) !} \times$

$J_{1}\left(n_{1}+l\right) J_{2}\left(n_{2}+k-\ell-1\right)$. 
4. If

$z(t)=$

$\frac{d^{m}}{d t^{m}} w(t) \int_{t_{0}}^{t} \frac{d^{n_{1}}}{d t^{n_{1}}} j_{1}(s) \frac{d^{n_{2}}}{d t^{n_{2}}} j_{2}(s) d s$ the $Z(k)=$

$\sum_{\ell=0}^{k} \sum_{s=0}^{k-\ell-1} \frac{(m+\ell) !\left(n_{1}+s\right) !\left(n_{2}+k-\ell-s-1\right) !}{(k-\ell) \ell ! s !(k-\ell-s-1) !} \times$

$J_{1}\left(n_{1}+s\right) J_{2}\left(n_{2}+k-\ell-s-\right.$

1) $W(m+\ell)$

\section{Fractional differential transform}

Let the anatomy and continuous function $z(t)$ in terms of a fractional reinforce series as follows:

$z(t)=\sum_{k=0}^{\infty} Z(k)\left(t-t_{0}\right)^{k / \alpha}$, (7)

where $\alpha$ is the order frction and $Z(k)$ is the frctional differential transform of $z(t)$.

The fractional derivative in Caputo is $D_{t_{0}}^{q} z(t)=$ $\frac{1}{\Gamma(m-q)} \frac{d^{m}}{d t^{m}}\left\{\int_{t_{0}}^{t}\left[\frac{z(s)-\sum_{k=0}^{m-1}\left(\frac{1}{k !}\right)\left(s-t_{0}\right)^{k_{Z}(k)}\left(t_{0}\right)}{(t-s)^{1+q-m}}\right]\right.$

The transformation of the initial conditions are defined as follows: $Z(k)=$ $\left\{\begin{array}{lc}\text { If } k / \alpha \in Z^{+}, & \frac{1}{(k / \alpha) !}\left[\frac{d^{k / \alpha} Z(t)}{d t^{k / \alpha}}\right]_{t=t_{0}} \\ \text { If } k / \alpha \notin Z^{+}, & 0\end{array}\right.$ ,(9)

where, $q$ is the order of fractional differential equation considered .

we succinct the fractional differential transform method with some theorems

\section{Theorem 4.1.}

1. If $h(t)=g(t) \pm f(t)$, then $H(k)=$ $G(k) \pm F(k)$.

2. If $h(t)=g(t) f(t)$, then $H(k)=$ $\sum_{l=0}^{k} G(l) F(k-l)$.

3. If

$h(t)=$

$f_{1}(t) f_{2}(t) \cdots f_{n-1}(t) f_{n}(t)$, then

$$
\begin{aligned}
& H(k)= \\
& \sum_{k_{n-1}=0}^{k} \sum_{k_{n-2}=0}^{k_{n-1}} \cdots \sum_{k_{2}=0}^{k_{3}} \sum_{k_{1}=0}^{k_{2}} F_{1}\left(k_{1}\right) F_{2}\left(k_{2}-\right. \\
& \left.k_{1}\right) \cdots F_{n-1}\left(k_{n-1}-k_{n-2}\right) F_{n}(k- \\
& \left.k_{n-1}\right)
\end{aligned}
$$

4. If $h(t)=\left(t-t_{0}\right)^{p}$, then $H(k)=$ $\delta(k-\alpha p)$ where ,

$$
\delta(k)=\left\{\begin{array}{l}
1 \text { if } k=0 \\
0 \text { if } k \neq 0
\end{array}\right.
$$

5. If $h(t)=D_{t_{0}}^{q}[f(t)]$, then $H(k)=$ $d s\} \frac{\Gamma(q+1+k / \alpha)}{\Gamma(1+k / \alpha)} F(k+\alpha q)$.

6. If

$h(t)=$

$\frac{d^{q_{1}}}{d t^{q_{1}}}\left[f_{1}(t)\right] \frac{d^{q_{2}}}{d t^{q_{2}}}\left[f_{2}(t)\right] \cdots \frac{d^{q_{n-1}}}{d t^{q_{n-1}}}\left[f_{n-1}(t)\right] \frac{d^{q_{n}}}{d t^{q_{n}}}\left[f_{n}(t)\right]$, then $H(k)=$

$\sum_{k_{n-1}=0}^{k} \sum_{k_{n-2}=0}^{k_{n-1}} \cdots \sum_{k_{2}=0}^{k_{3}} \sum_{k_{1}=0}^{k_{2}} \frac{\Gamma\left(q_{1}+1+k_{1} / \alpha\right)}{\Gamma\left(1+k_{1} / \alpha\right)} \frac{\Gamma\left[q_{2}+1+\left(k_{2}-k_{1}\right) / \alpha\right]}{\Gamma\left[1+\left(k_{2}-k_{1}\right) / \alpha\right]} \cdots \frac{\Gamma\left[q_{n-1}+1+\left(k_{n-1}-k_{n-2}\right) / \alpha\right]}{\Gamma\left[1+\left(k_{n-1}-k_{n-2}\right) / \alpha\right]}$ $\left.\alpha q_{1}\right) \times F_{2}\left(k_{2}-k_{1}+\alpha q_{2}\right) \cdots F_{n-1}\left(k_{n-1}-k_{n-2}+\alpha q_{n-1}\right) \times$ $F_{n}\left(k-k_{n-1}+\alpha q_{n}\right)$, where $\alpha q_{i} \in Z^{+}$for $i=1,2, \cdots, n$. for $k=0,1, \ldots, q \alpha$

\section{Numerical examples}

Example 1 To solve the equation

$$
\begin{aligned}
& z^{q}(t)=\frac{1}{2} z^{\prime}(t) u(t)-u(t)- \\
& \int_{0}^{t}\left[z^{\prime}(s)\right]^{2} d s+\frac{1}{2}+t, \quad t \geq 0
\end{aligned}
$$


with conditions

$z(0)=-1, \quad z^{\prime}(0)=1, \quad z^{\prime \prime}(0)=$ $\frac{1}{2}$

By using differential transformation method on Equ.(10), for $k=1,2, \ldots$, we acquire

$Z(k+\alpha q)=\frac{\Gamma(1+k / \alpha)}{\Gamma(\mathrm{q}+1+k / \alpha)}\left[\frac{1}{2} \sum_{\ell=0}^{k}(\ell+\right.$ 1) $Z(\ell+1) Z(k-\ell)-Z(k)-$ $\frac{1}{k} \sum_{\ell=0}^{k-1}(\ell+1)(k-\ell) Z(\ell+1) Z(k-$ $\left.\ell)+\frac{1}{2} \delta(k)+\delta(k-1)\right]$,

where $\alpha$ is the unknown value of the fraction of $q$.

By using Eq.(9) the initial conditions is

$Z(0)=-1$

$Z(1)=1$

$Z(2)=\frac{1}{2}$

$Z(3)=0$,

for $k=3, \ldots, 9,11,12, \ldots, 19,21$

$Z(10)=1$

$Z(20)=\frac{1}{4}$

Now, in [1 ], when $q=2$, the exact solution of Eq.(10) is $(z(t)=$ $\sin (t)-\cos (t))$ and it's got from the serise solution

$$
\begin{aligned}
z(t)=-1+t & +\frac{1}{2} t^{2}-\frac{1}{6} t^{3}-\frac{1}{24} t^{4} \\
& +\frac{1}{120} t^{5}+\frac{1}{720} t^{6} \\
& -\frac{1}{5040} t^{7}+\cdots
\end{aligned}
$$

Here, we take $q=2.1$ then the approximate solution for Eq.(10) is

$z(t)=-1+t^{\frac{1}{10}}+\frac{1}{2} t^{\frac{2}{10}}+t+\frac{1}{4} t^{2}-$ $2 \frac{\Gamma\left(\frac{11}{10}\right)}{\Gamma\left(\frac{32}{10}\right)} t^{\frac{11}{5}}+\frac{1}{4} \frac{\Gamma\left(\frac{12}{10}\right)}{\Gamma\left(\frac{33}{10}\right)} t^{\frac{23}{10}}-\frac{1}{12} \frac{\Gamma\left(\frac{13}{10}\right)}{\Gamma\left(\frac{34}{10}\right)} t^{\frac{24}{10}}$ $+\ldots$

Fig.1 shows the complete solution for Eq.(10), when $q=2$, Fig. 2 shows the Sacrificial solution for Eq.(10), when $q=2.1$

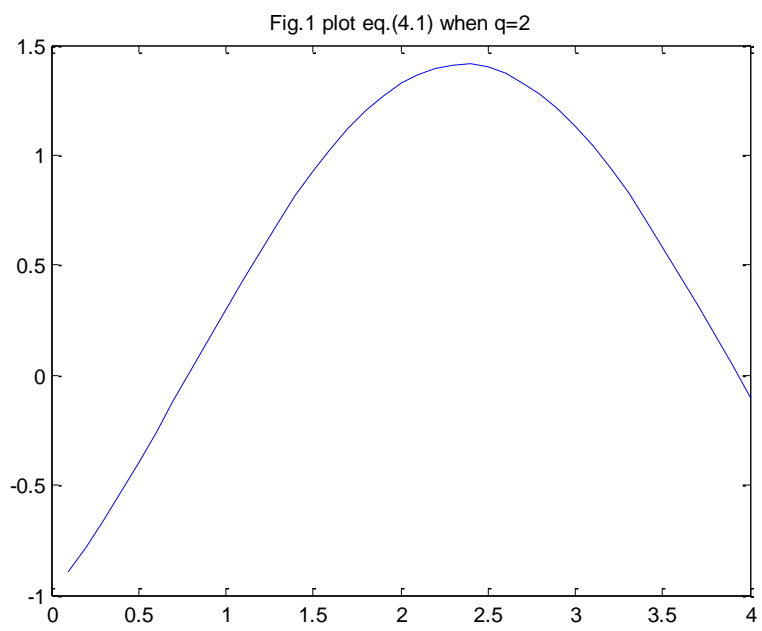




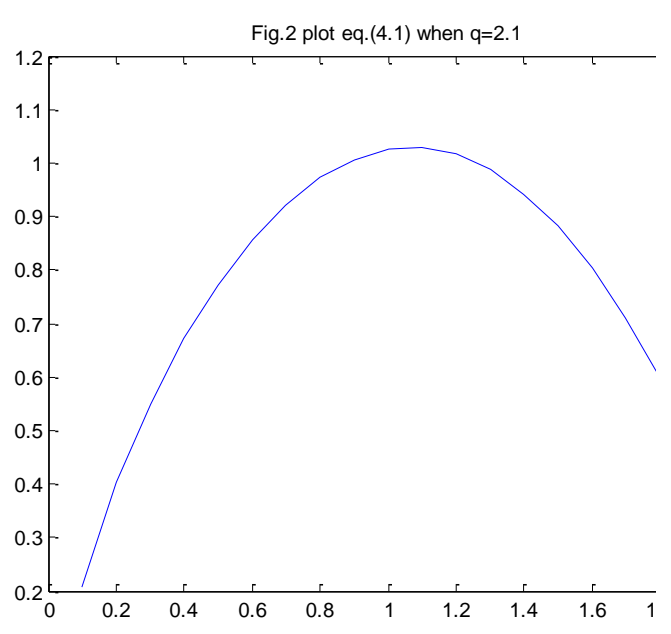

Example 2. To solve the equation

$$
\begin{aligned}
& z^{q}(t)= \\
& \frac{1}{2} z^{\prime}(t)-z(t) \int_{0}^{t} z^{\prime}(s) z^{\prime}(s) d s+\frac{1}{2} e^{3 t}
\end{aligned}
$$

with conditions

$$
\mathrm{z}(0)=\mathrm{z}^{\prime}(0)=1
$$

By using differential transformation method on Eq.(14), for $k=1,2, \ldots$, we acquire

$Z(k+19)=\frac{\Gamma(1+k / 10)}{\Gamma(q+1+k / 10)}\left[\frac{k+1}{2} Z(k+\right.$ 1) -

$\sum_{\ell=0}^{k-1} \sum_{s=0}^{k-\ell-1} \frac{(k-\ell-s)(s+1)}{k-\ell} Z(\ell) Z(s+$ 1) $\left.Z(k-\ell-s)+\frac{3^{k}}{2 k !}\right]$,

where $\alpha$ is the unknown value of the fraction of $q$.

By using Eq.(9) the initil conditions is $Z(0)=1$
$Z(1)=1$

$Z(3)=0$, for $k=$

$2,3, \ldots, 9,11,12, \ldots, 19$

$$
Z(10)=1
$$

Now, in [1 ], when $q=2$, the the exact solution of Eq.(14) is $(z(t)=$ $\left.e^{t}\right)$ and it's got from the series solution

$$
\begin{aligned}
z(t)=1+t+ & \frac{1}{2} t^{2}+\frac{1}{6} t^{3}+\frac{1}{24} t^{4} \\
& +\frac{1}{120} t^{5}+\frac{1}{720} t^{6} \\
& +\frac{1}{5040} t^{7}+\cdots
\end{aligned}
$$

If we continues for $k>5$ the solution is $z(t)=e^{t}$

Here, we take $q=2.1$ then the approximate solution for Eq.(14) is

$z(t)=1+t^{\frac{1}{10}}+t+\frac{1}{2} \frac{\Gamma\left(\frac{11}{10}\right)}{\Gamma\left(\frac{30}{10}\right)} t^{\frac{20}{10}}+$ $\frac{5}{4} \frac{\Gamma\left(\frac{12}{10}\right)}{\Gamma\left(\frac{31}{10}\right)} t \frac{21}{10}+\frac{9}{4} \frac{\Gamma\left(\frac{13}{10}\right)}{\Gamma\left(\frac{32}{10}\right)} t^{\frac{22}{10}}+\cdots$

Fig.3 shows the complete solution for Eq.(14) acquired for the value of $q=$ 2, i.e $\left(z(t)=e^{t}\right)$. Fig.4 shows the Sacrificial solution for eq.(14) acquired for the value of $q=1.9$. 


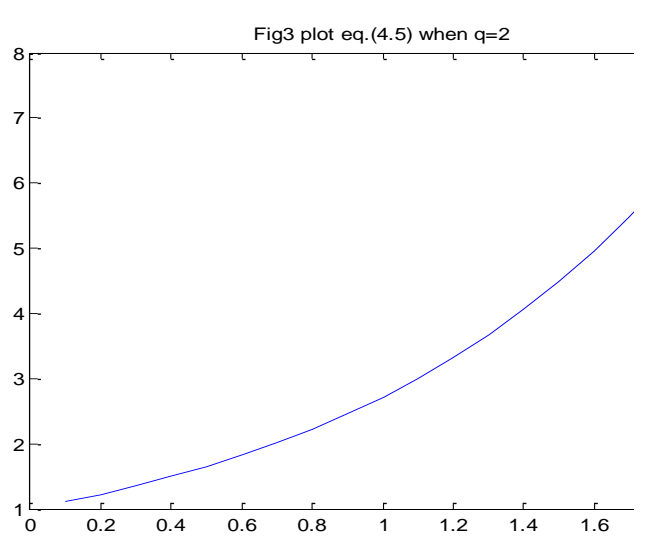

By practise differential transformation method on Eq.(18),for $k=1,2, \ldots$, we acquired

$Z(k+\alpha q)=\frac{\Gamma(1+k / \alpha)}{\Gamma(\mathrm{q}+1+k / \alpha)}[(k+$

1) $Z(k+1)-$

$2 \sum_{\ell=0}^{k-1} \sum_{s=0}^{k-\ell-1} Z(\ell) Z(s) Z(k-\ell-$

$\left.s)+\frac{1}{k !} \sum_{\ell=0}^{k} \frac{\delta(\ell-3) 3^{k-\ell}}{(k-\ell) !}\right]$,

where $\alpha$ is the obscure value of the fraction of $q$.

Initial conditions in

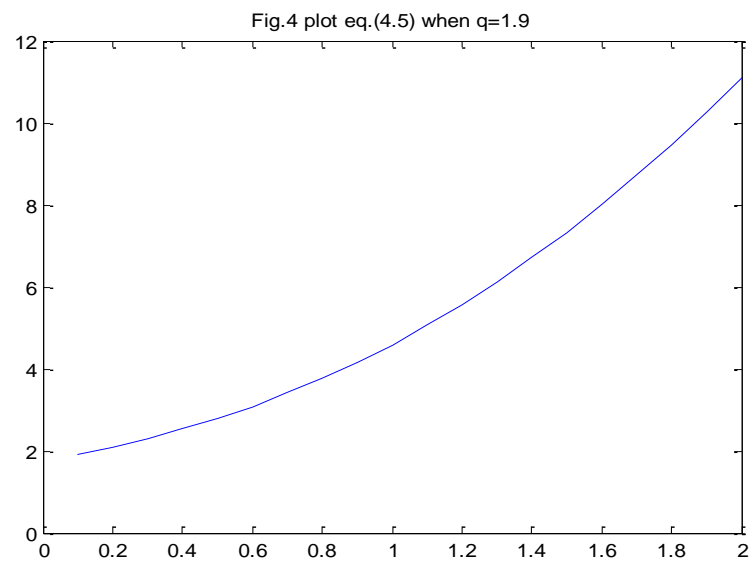

Eq.(19)are transformed by employment Eq.(9) as follows:

$$
\begin{aligned}
& Z(0)=0 \\
& Z(1)=-1 \\
& Z(2)=0, \text { for } k= \\
& 2,3, \ldots, 9,11,12, \ldots, 18 \\
& Z(10)=1,
\end{aligned}
$$

Example 3 we take the D.T. for the

Now, in [1 ], when $q=2$, the the exact solution of Eq.(18) is $\left(z(t)=t e^{t}\right)$ and it's got from the series solution

following integro-differential equation

$z^{q}(t)=$

$z^{\prime}(t)-2 z(t) \int_{0}^{t} z(s) z^{\prime}(s) d s+e^{t}+$

$t^{3} e^{3 t}$

with initial conditions

$z(0)=0, \quad z^{\prime}(0)=1$

(19)

$$
\begin{aligned}
z(t)=t+t^{2} & +\frac{1}{2} t^{3}+\frac{1}{6} t^{4}-\frac{1}{24} t^{5} \\
& +\frac{1}{120} t^{6}-\frac{1}{720} t^{7}+\cdots
\end{aligned}
$$

If we continues for $k>5$ the solution is $z(t)=t e^{t}$

when, $q=1.8$ the approximate solution for eq.(18) is 
$z(t)=t^{\frac{1}{10}}+t+\frac{\Gamma\left(\frac{11}{10}\right)}{\Gamma\left(\frac{29}{10}\right)} t^{\frac{19}{10}}+$

$\frac{1}{2} \frac{\Gamma\left(\frac{12}{10}\right)}{\Gamma\left(\frac{30}{10}\right)} t^{\frac{20}{10}}+\frac{7}{6} \frac{\Gamma\left(\frac{13}{10}\right)}{\Gamma\left(\frac{31}{10}\right)} t^{\frac{21}{10}}+\cdots$

Fig. 5 shows the complete solution for Eq.(18) when $q=2$. Fig. 6 shows the Sacrificial solution for eq.(18) acquired for the value of $q=1.8$.
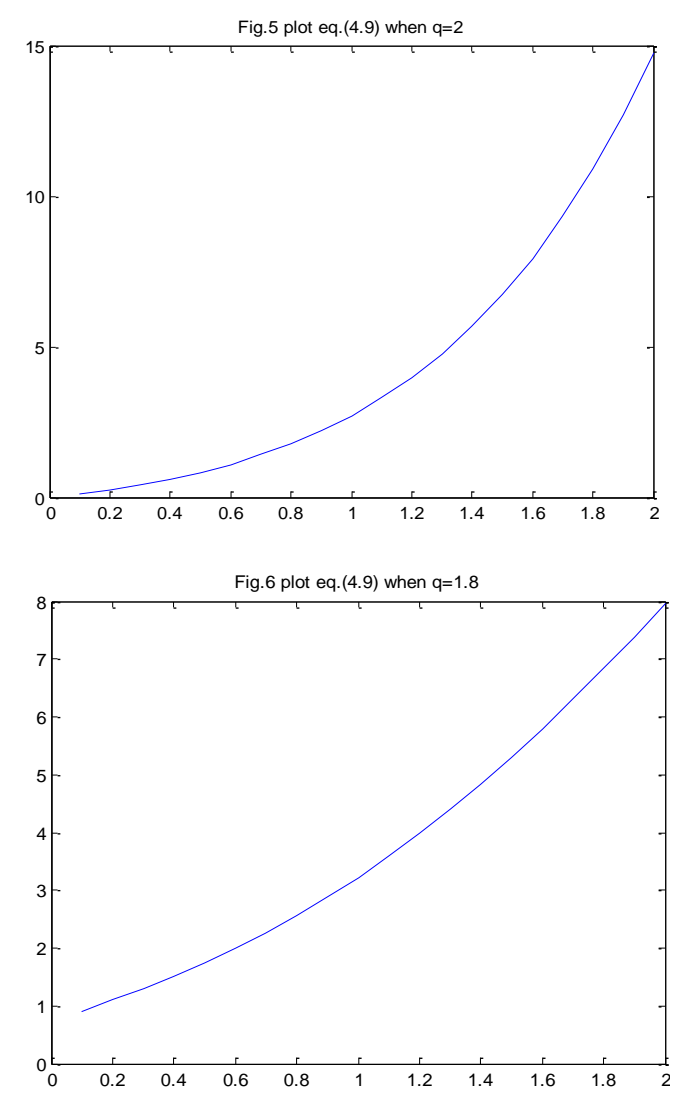

2. M.Mohseni Moghadam and H.Saeedi , Application of differential transforms for solving the volterra integro-partial differential equations, Shiraz University , 2010.

3. Vedat suat Ertürk, Shaher Momani, Solving systems of fractional differential equations using differential transform method, Journal of Computational and Applied Mathematics ,2008.

4. Saurabh M.,Akshay B. and Prashikdivya G. , Solution of nonlinear differential transform method, IOSR-JM , 2014.

\section{$\underline{\text { References }}$}

1. A.Borhanifar, Reza Abazari,

Differential transform method for a class of nonlinear integro-differential equations with derivative type kernel, Canadian Journal on Computing in Mathematics, 2012. 